\title{
La transformada de Fourier y su aplicación en el procesamiento de imágenes, Quito, Ecuador, Diciembre del 2008
}

$$
F(u, v)=\sum_{n=0}^{N-1} \sum_{m=0}^{M-1} f(n, m) e^{-j 2 \pi\left(\frac{u n}{N}+\frac{v m}{m}\right)}
$$

\section{Autores:}

Matemático René Mauricio García Oquendo Ing. Gabriel Enrique Pazmiño Castillo 


\section{Resumen}

El objeto de estudio es la Transformada de Fourier y su aplicación al procesamiento digital de imágenes, para lo cual se considera la Transformada Discreta de Fourier y su algoritmo eficiente de cálculo denominado Transformada Rápida de Fourier. La Transformada Discreta de Fourier es una herramienta que permite transformar las señales del dominio espacial o temporal al de frecuencias, permitiendo analizarlas desde el punto de vista de sus componentes de frecuencia. El proyecto hace referencia a la investigación sobre los fundamentos matemáticos, algoritmos y aplicación práctica en el procesamiento de imágenes de la Transformada de Fourier, y la difusión a través de la elaboración de un texto orientado a los estudiantes de Informática, Sistemas o ramas afines, ya que la implementación de la (más conocida como FFT por sus siglas en inglés) aplicada al procesamiento de imágenes, ya que en la bibliografía especializada, no se encuentra explícitamente bien documentada. Durante el desarrollo del proyecto fue necesario revisar y estructurar de manera didáctica los fundamentos matemáticos de la Transformada Rápida de Fourier y explicar sus algoritmos mediante programas claramente documentados y con resultados de prueba. Para la aplicación de la transformada Rápida de Fourier al procesamiento de imágenes, se hace una revisión de la convolución, el diseño de filtros y su aplicación a la restauración de imágenes, explicando los procesos y documentando los programas que soportan a dichos procesos. Como está estructurado el proyecto, logra reunir en unúnico documento, poruna partela base matemática, los algoritmos y procesos implementados mediante programas claramente explicados, de tal manera que sirve de referencia para el estudio, implementación y aplicación de la Transformada Rápida de Fourier en el procesamiento digital de imágenes.

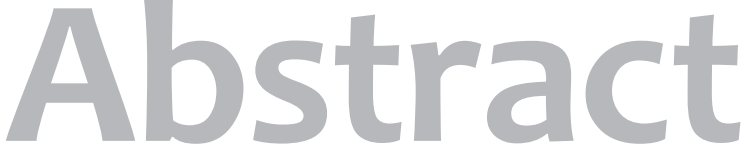

The purpose of this research was to study the Fourier transform and its application to digital image processing, for this, the Discrete Fourier Transform and its efficient calculation algorithm called Fast Fourier Transform was considered. The Discrete Fourier Transform is a tool that allows transform signals from the spatial or temporal domain to frequency domain, allowing analysis in terms of their frequency components.

The project refers to research on the mathematical foundations, algorithms and practical applications in image processing of the Fourier transform, and diffusion through the development of a text designed for students of Computer Systems or related branches, because the implementation of the Fast Fourier Transform (better known as FFT stands for English) applied to image processing, in the specialized literature, is not explicitly documented. During the development of the project was necessary to review and structuring in didactic manner the mathematical foundations of the Fast Fourier Transform, explain their algorithms and clearly document the programs with test results.

For the application of the fast Fourier transform to image processing, a review of the convolution, filter design and its application to image restoration, explaining processes and documenting programs that support these processes was made. As the project is structured, brings together in one document the mathematical foundation of the Fast Fourier Transform, algorithms implemented through programs and processes clearly explained, so that serves as a reference for the study, implementation and application of the Fast Fourier Transform in digital image processing. 


\section{Introducción}

La Transformada de Fourier es una herramienta matemática, que en su versión discreta se conoce como Transformada Discreta de Fourier, que ha sido ampliamente usado en el tratamiento digital de señales, y se encuentra implementada bajo la forma de dispositivos electrónicos de reconocimiento de voz e imagen; puede ser aplicada a varios campos como el análisis espectral, las ecuaciones diferenciales, la resolución de problemas elásticos estacionarios y dinámicos, entre otras.

El presente trabajo, presenta una relación con los aspectos teóricos, con la aplicación práctica de la Transformada de Fourier en el procesamiento digital de imágenes mediante el desarrollo de aplicaciones que implementan los algoritmos de la Transformada Rápida de Fourier, los mismos que son explicados y analizados de una manera clara y didáctica, en un texto de nivel superior orientado a los estudiantes de Informática, Sistemas y Ciencias de la Computación.

\section{- Importancia}

El desarrollo matemático de la transformada de Fourier fue explicado por Jean Baptiste Joseph Fourier, en su libro la Teoría Analítica del Calor, publicado en 1822; posteriormente, en 1965 Cooley y Tukey publicaron su artículo "Un algoritmo para calcular las Series de Fourier Complejas", el cual es conocido como algoritmo FFT (Fast Fourier Transform), y, que con el desarrollo acelerado de las computadoras digitales ha permitido la aplicación de la FFT a diferentes campos tales como mecánica aplicada, acústica, biomedicina, métodos numéricos, instrumentación, electromagnetismo, etc., lo que señala la importancia de su aplicación en el actual desarrollo tecnológico estas ramas y que sin el uso de la FFT no se hubiera logrado, ya que todas ellas tienen como denominador común el tratamiento digital de señales en el que se hace un uso intensivo de la FFT.
Su aplicación al procesamiento de imágenes se encuentra documentado en los libros y textos específicos sobre la materia a un nivel teórico, en los que no se expone directamente, la forma de implementación de los diferentes algoritmos, y en el mejor de los casos presentan una descripción narrativa del algoritmo, como ejemplo se puede revisar el libro Digital Image Processing de González y Woods, 3ra. Edición. Por otra parte, los libros específicos sobre la Transformada Rápida de Fourier, se centran suaplicación mayormente a la fundamentación matemática y explicación de los algoritmos, presentando aplicaciones más orientadas al Procesamiento Digital de Señales, que corresponde al campo de la Electrónica.

\section{- Objetivo del estudio}

Con las consideraciones anteriores, el presente proyecto, reúne en un documento la fundamentación matemática, los algoritmos de la transformada Rápida de Fourier, y la aplicación de los mismos al procesamiento de imágenes mediante el desarrollo de software que muestra como se implementan dichos algoritmos.

\section{Materiales, Métodos y Procedimientos}

\section{- Diseño de Investigación}

Para el desarrollo de la investigación se aplicó el método lógico deductivo, el cual permitió desarrollar y explicar los fundamentos matemáticos de la FFT, también se utilizó el método experimental, para comprobar los resultados arrojados por los algoritmos implementados al aplicarlos en las imágenes digitales.

\section{- Materiales}

Para la realización del proyecto fue necesario identificar la bibliografía especializada en dos áreas: transformada Rápida de Fourier y Procesamiento Digital de Imágenes. 


\section{- Procedimientos}

La investigación se dividió en tres fases:

- Fundamentos matemáticos de la Transformada de Fourier

- Análisis de los algoritmos de la Transformada Rápida de Fourier

- Análisis, desarrollo e implementación de Aplicaciones utilizando la transformada de Fourier.

\section{- Fundamentos matemáticos:}

En esta fase se estudió y analizó los fundamentos matemáticos de la Transformada de Fourier, para lo cual se partió de las series de Fourier, llegando hasta la Integral de Fourier; y de acuerdo con los objetivos del proyecto, se desarrolló en MatLab la aplicación que permite mostrar en forma didáctica el cálculo de los coeficientes de Fourier para series periódicas, así como su transformada.

Para el desarrollo de esta aplicación fue necesario estudiar el desarrollo de interfaces gráficas con el objeto de visualizar los resultados de la implementación algorítmica de la serie de Fourier, el gráfico presentado a continuación muestra la pantalla principal de la aplicación, y cuyo código fuente, debidamente explicado forma parte del documento de la investigación.
Figura No 1

Algoritmos de la Transformada Rápida de Fourier

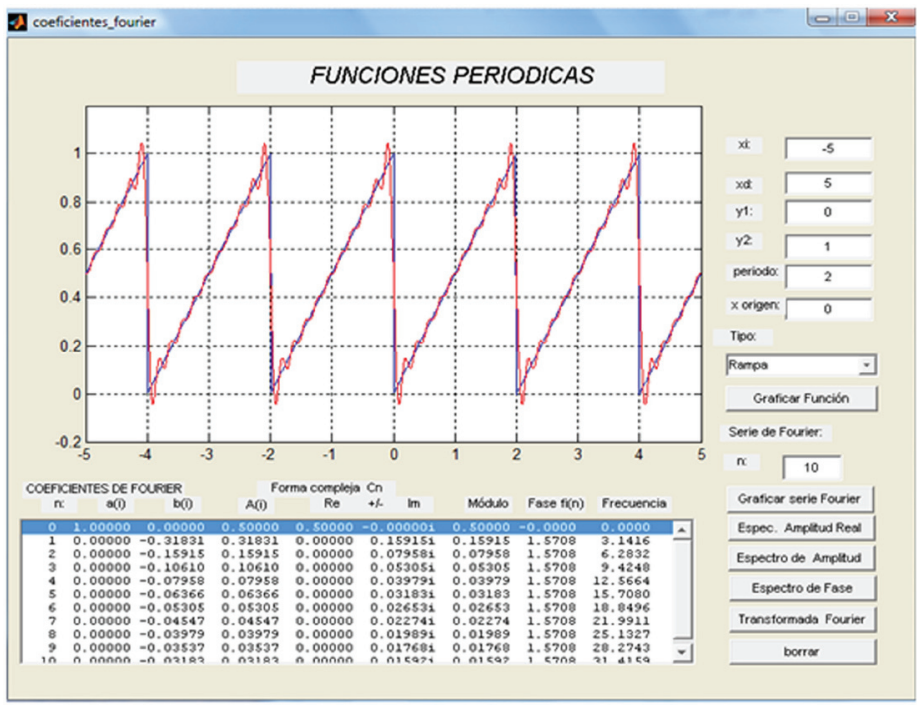

La transformada discreta de Fourier en una dimensión está dada por:

$$
X(u)=\sum_{n=0}^{N-1} x(n) e^{\frac{-j 2 \pi u n}{N}}
$$

Donde $\quad x(n)$ es el conjunto de datos original

$X(u)$ es la transformada de $x(n)$

$\mathrm{N}$ es el número de elementos

u representa la variable en el dominio de la frecuencia

Y su extensión a dos dimensiones está definida por:

$$
F(u, v)=\sum_{n=0}^{N-1} \sum_{m=0}^{M-1} f(n, m) e^{-j 2 \pi\left(\frac{u n}{N}+\frac{v m}{m}\right)}
$$


Partiendo de estas definiciones se analizaron los algoritmos en diferentes fuentes bibliográficas y se implementaron en Visual Basic.Net los siguientes:

\section{- Para una dimensión}

- Método directo: el cual implementa directamente la definición de la transformada discreta en una dimensión.

- Método recursivo: el cual está basado en el algoritmo FFT propuesto por Cooley y Tukey.

- Método iterativo, también basado en el algoritmo anterior, y que presenta una mayor complejidad, puesto que requiere de la implementación del algoritmo de reversión de bits de un arreglo.

\section{Figura No 2}

\section{El prototipo de esta implementación se muestra a continuación}

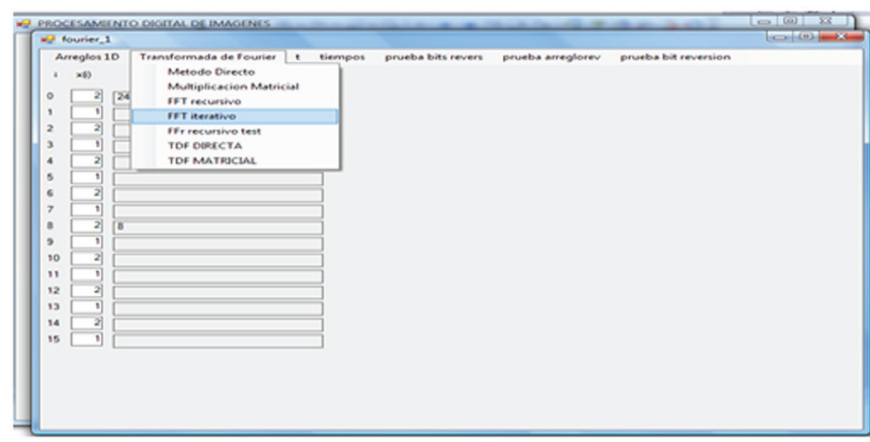

Para la creación de los prototipos, además de los algoritmos implementados fue necesario crear el conjunto de funciones $y$ procedimientos que trabajan con números complejos.

Para la comprobación de los resultados, se realizaron varias corridas con diferentes tamaños de arreglos, las cuales fueron contrastadas con los datos arrojados por la herramienta Análisis de Fourier que implementa Excel. La implementación de la Transformada discreta bidimensional necesitó el análisis y programación de los siguientes procedimientos:

Transformada directa bidimensional, la cual utiliza la fórmula de la transformada discreta bidimensional de Fourier, y cuyos resultados sirvieron para contrastar los datos devueltos por otros métodos.

FFT-2D, el mismo que implementa la transformada rápida de Fourier en dos dimensiones, utilizando como base la implementación en una dimensión. El prototipo de esta implementación se muestra a continuación:

\section{Figura No 3}

\section{Implementación de las aplicaciones de la FFT}

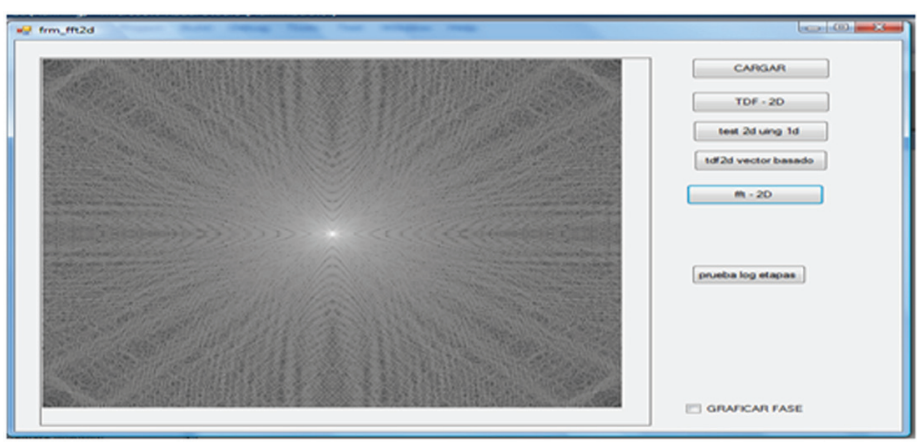

En el estado actual del proyecto, se está analizando las aplicaciones de la FFT en el procesamiento digital de imágenes previo a la construcción del software correspondiente, así como la respectiva documentación explicativa que se integrará al texto.

\section{Resultados y Discusión}

Los principales logros alcanzados en la realización del proyecto hasta el momento fueron:

1. Unificar en un documento la fundamentación matemática de la Transformada de Fourier, la comprensión de los algoritmos 
que permiten su implementación tanto en una como en dos dimensiones mediante software cuyo código fuente es parte del presente trabajo.

2. Contar conbibliografía avanzada en el tema dela investigación. Mediante este proyecto fue posible realizar la importación de libros de reciente publicación y de diferentes niveles de complejidad en las áreas de tratamiento de imágenes y de la Transformada de Fourier, los cuales sirven para el desarrollo de futuros proyectos en diferentes áreas como sería la del tratamiento de imágenes médicas;

Cabe destacar los siguientes aspectos que durante el desarrollo del proyecto constituyen elementos de interés:

- El desarrollo de la fundamentación matemática de la Transformada de Fourier requiere conocimientos de cálculo integral y números complejos.

- La comprensión de los algoritmos involucrados para la implementación de la Transformada Rápida de Fourier, requiere conocer las herramientas matemáticas que subyacen detrás de los mismos, y un buen nivel de programación en algún lenguaje.

- Es importante que quienes estudian la FFT, a efectos de poder realizar diferentes simulaciones tengan conocimiento de MatLab para poder visualizar gráficamente los resultados.

- La revisión de la bibliografía adquirida permitió ver las tendencias actuales en cuanto al procesamiento digital de imágenes, y demuestra que en los libros especializados de procesamiento de imágenes no se encuentran implementaciones prácticas que de carácter didáctico demuestren el uso de la FFT, por lo que es necesario recurrir a la bibliografía especializada sobre FFT, en los que si se encuentra la forma de implementar la FFT en lenguajes de programación como FORTRAN, C o BASIC, pero mencionan muy brevemente o casi nada su aplicación al procesamiento de imágenes.

\section{Conclusiones y Recomendaciones}

Específicamente, mediante el desarrollo de los prototipos antes señalados, se demostró, coincidiendo con todos los autores, como Gonzalez, Brigman, Burger, etc.:

1. Que la implementación directa de la Transformada Discreta de Fourier para un conjunto de $\mathrm{N}$ elementos, es de orden $\mathrm{N}^{2}$, lo que la hace impracticable para el manejo de imágenes mayores a 100 x 100 pixeles, y que la mejor alternativa de implementación constituyen los algoritmos de FFT, los cuales están basados en el de Cooley y Tukey y cuyo orden es de $\mathrm{N} \log _{2} \mathrm{~N}$ y que reducen significativamente el tiempo de uso del procesador.

2. Los algoritmos que implementan la FFT, y que se encuentran en la bibliografía especializada, tienen un alto nivel de optimización, y son el resultado de los esfuerzos de varios investigadores durante las últimas décadas, lo que ha permitido el uso práctico de la Transformada de Fourier en diferentes áreas. 


\section{Bibliografía consultada}

1. Brigham, E. (1988). Fast Fourier Transform and Its Applications.

New York. Prentice Hall

2. Burger, W. James. (2007). Digital Image Processing: An Algorithmic Introduction using Java. EE.UU.: Springer

3. Gonzalez, C., Woods, R. (2008). Digital Image Processing (3ra. Ed). New Jersey. EE.UU: Prentice Hall

4. James, J. (2003). Student's Guide to Fourier Transforms. EE.UU. Cambridge University Press

5. James, G. (2002). Matemáticas avanzadas para ingeniería. EE.UU... Prentice Hall

6. Russ, J. (2006). The Image Processing Handbook, (5ta. Ed). EE.UU. CRC Press

- El Investigador

Ing. Gabriel Pazmiño Castillo

Ingeniero Civil, Universidad Tecnológica Equinoccial, 1986.

\section{El Investigador}

Ing. Gabriel Pazmiño Castillo

\section{Ingeniero Civil; Universidad} Tecnológica Equinoccial, 1986

\section{Anexo}

\begin{tabular}{|c|c|c|}
\hline TÍTULO & AUTOR & ÁREA \\
\hline Digital Image Processing (3rd Edition) & \begin{tabular}{|l|} 
Rafael C. Gonzalez \\
(Author), Richard E. Woods \\
(Author)
\end{tabular} & PDI \\
\hline $\begin{array}{l}\text { Algorithms for Image Processing and } \\
\text { Computer Vision }\end{array}$ & J. R. Parker (Author) & PDI \\
\hline $\begin{array}{l}\text { Practical Algorithms for Image } \\
\text { Analysis with CD-ROM }\end{array}$ & \begin{tabular}{|l|} 
Lawrence O'Gorman \\
(Author), Michael J. \\
Sammon (Author), Michael \\
Seul (Author)
\end{tabular} & PDI \\
\hline $\begin{array}{l}\text { The Pocket Handbook of Image } \\
\text { Processing Algorithms In C }\end{array}$ & $\begin{array}{l}\text { Harley R. Myler and Arthur } \\
\text { R. Weeks }\end{array}$ & PDI \\
\hline $\begin{array}{l}\text { The Image Processing Handbook, } \\
\text { Fifth Edition (Image Processing } \\
\text { Handbook) }\end{array}$ & John C. Russ & PDI \\
\hline $\begin{array}{l}\text { Foundations of Image Science } \\
\text { (Hardcover) }\end{array}$ & $\begin{array}{l}\text { Harrison H. Barrett } \\
\text { (Author), Kyle Myers } \\
\text { (Author) }\end{array}$ & PDI \\
\hline $\begin{array}{l}\text { Digital Image Processing: An } \\
\text { Algorithmic Introduction using Java } \\
\text { (Hardcover) }\end{array}$ & $\begin{array}{l}\text { Wilhelm Burger (Author), } \\
\text { Mark James Burge (Author) }\end{array}$ & PDI \\
\hline $\begin{array}{l}\text { Introduction to Image Processing and } \\
\text { Analysis (Hardcover) }\end{array}$ & $\begin{array}{l}\text { John C. Russ (Author), J. } \\
\text { Christian Russ (Author) }\end{array}$ & PDI \\
\hline $\begin{array}{l}\text { Computer Vision: A Modern } \\
\text { Approach (Hardcover) }\end{array}$ & $\begin{array}{l}\text { David A. Forsyth (Author), } \\
\text { Jean Ponce (Author) }\end{array}$ & PDI \\
\hline $\begin{array}{l}\text { A Student's Guide to Fourier } \\
\text { Transforms (Paperback) }\end{array}$ & J. F. James (Author) & \begin{tabular}{|l} 
Transformada de \\
Fourier
\end{tabular} \\
\hline $\begin{array}{l}\text { Fast Fourier Transform and Its } \\
\text { Applications }\end{array}$ & E. Brigham (Author) & \begin{tabular}{|l} 
Transformada de \\
Fourier
\end{tabular} \\
\hline $\begin{array}{l}\text { Machine Vision : Theory, Algorithms, } \\
\text { Practicalities }\end{array}$ & E. R. Davies (Author) & PDI \\
\hline $\begin{array}{l}\text { Understanding the FFT, Second } \\
\text { Edition, Revised (Paperback) }\end{array}$ & Anders E. Zonst (Author) & $\begin{array}{l}\text { Transformada de } \\
\text { Fourier }\end{array}$ \\
\hline $\begin{array}{l}\text { Fourier Transform and Its } \\
\text { Applications (Paperback) }\end{array}$ & Ronald Bracewell & $\begin{array}{l}\text { Transformada de } \\
\text { Fourier }\end{array}$ \\
\hline $\begin{array}{l}\text { Digital Signal Processing Algorithms: } \\
\text { Number Theory, Convolution, Fast } \\
\text { Fourier Transforms, and Applications } \\
\text { (Crc Press Computer Engineering } \\
\text { Series) (Hardcover) }\end{array}$ & Hari Krishna (Author) & $\begin{array}{l}\text { Tratamiento de } \\
\text { señales }\end{array}$ \\
\hline $\begin{array}{l}\text { Fast Fourier Transforms: Second } \\
\text { Edition (Studies in Advanced } \\
\text { Mathematics) (Hardcover) }\end{array}$ & James S. Walker & \begin{tabular}{|l} 
Transformada de \\
Fourier
\end{tabular} \\
\hline $\begin{array}{l}\text { Handbook of Image and Video } \\
\text { Processing }\end{array}$ & by Alan C. Bovik (Author) & PDI \\
\hline
\end{tabular}

Detalle de bibliografía adquirida 